\title{
Efficiency Enhancement of Axial Junction InP Single Nanowire Solar Cells by Dielectric Coating
}

Zhiqin Zhong ${ }^{1, a, b}$, Ziyuan Li 1,a , Qian Gao ${ }^{\text {a }}$, Zhe $\mathrm{Li}^{\mathrm{c}}$, Kun Peng a ${ }^{\text {a }}$ Li Li ${ }^{\text {d }}$, Sudha Mokkapati a Kaushal Vora ${ }^{\mathrm{d}}$, Jiang Wu ${ }^{\text {bee }}$, Guojun Zhang ${ }^{\mathrm{b}}$, Zhiming Wang ${ }^{\mathrm{b}}$, Lan Fu ${ }^{\mathrm{a}, *}$, Hark Hoe Tan ${ }^{\mathrm{a}}$, and Chennupati Jagadish ${ }^{\text {a }}$

${ }^{1}$ Equal first author

Z. Q. Zhong and Z. Y. Li are equal first author.

*Corresponding author

E-mail address: lan.fu@anu.edu.au (L. Fu).

${ }^{a}$ Department of Electronic Materials Engineering, Research School of Physics and Engineering, The Australian National University, Canberra, ACT 2601, Australia

${ }^{b}$ Institute of Fundamental and Frontier Science, State Key Laboratory of Electronic Thin Films and Integrated Devices, University of Electronic Science and Technology of China, Chengdu, 610054, China

${ }^{c}$ Department of Applied Mathematics, Research School of Physics and Engineering, The Australian National University, Canberra, ACT 2601, Australia

${ }^{d}$ Australian National Fabrication Facility, Department of Electronic Materials Engineering, Research School of Physics and Engineering, The Australian National University, Canberra, ACT 2601, Australia

${ }^{e}$ Department of Electronic and Electrical Engineering, University College London, London WCIE 7JE, UK

Keywords: III-V semiconductors, nanowire solar cells, axial junction, selective-area MOVPE, EBIC, dielectric coating

Abstract: In this work we demonstrate single axial p-i-n junction InP nanowire (NW) solar cells grown by selective-area metal organic vapor phase epitaxy (SA-MOVPE) technique. A power conversion efficiency of up to $6.5 \%$ was realized in the single NW solar cell (horizontally lying on substrate) without any surface passivation. Electron beam induced current (EBIC) and photocurrent mapping were performed to investigate the electrical properties of the NW solar cells and their influence on device performance, which are essential for an in-depth understanding of the design requirements for NW solar cells. A further conformal $\mathrm{SiN}_{\mathrm{x}}$ layer was deposited on the single NW solar cell devices by plasma-enhanced chemical vapor deposition (PECVD). Overall efficiency improvement has been obtained in the $\mathrm{SiN}_{\mathrm{x}}$-coated devices with a remarkable up to $62 \%$ increase to a peak efficiency of $10.5 \%$, which to our knowledge is the highest efficiency reported for horizontal single NW solar cells. This has been 
attributed to an enhanced optical antenna effect and effective surface passivation due to $\mathrm{SiN}_{\mathrm{x}}$ coating, as respectively confirmed by numerical simulation and time-resolved photoluminescence (TRPL) measurements. Our work demonstrates that dielectric coating is a promising simple approach to achieve high performance III-V NW solar cells. 


\section{Introduction}

III-V semiconductor nanowires (NWs) devices have received increasing attention in recent years as promising multi-functional building blocks for future highly compact optoelectronic/photonic integrated circuits due to their superior optical (direct band gap and thus high absorption coefficient) and electrical (high carrier mobility) properties. To date, various optoelectronic/photovoltaic devices based on III-V semiconductor NWs have been demonstrated, such as lasers [1, 2], light-emitting diodes [3, 4], photodetectors [5] and solar cells [6, 7]. For solar cell applications, researchers endeavor to achieve higher efficiencies at lower cost. To date record efficiencies of $13.8 \%$ and $15.3 \%$ have been reported in InP [6] and GaAs [8] nanowire array solar cells respectively. These results show great promise to reduce manufacture cost by achieving high solar cell efficiency while largely reducing material consumption [9-14]. However to date, the experimentally reported efficiency is still far below theoretical predictions [15], due to the challenges in design, characterization, control and optimization of various important issues such as light absorption, carrier transport and collection (via p-n junction), material growth, and device fabrication for these nanoscale non-planar structure devices.

To obtain optimal energy-conversion efficiencies for nanowire solar cells (NWSCs), it is essential to understand the individual NW photovoltaic performance. Single NW solar cells have been investigated in both vertical configuration (incident light propagating along the direction parallel to the axis of the nanowire) [16] and horizontal configuration (incident light propagating along the direction perpendicular to the axis of the nanowire) [17] In both configurations, the incident light couples to different resonant modes leading to different absorption characteristics of nanowires from the same material in bulk, planar configuration [18]. The localized twodimensional resonant modes are able to give rise to a light-trapping optical antenna effect and a substantially increased short-circuit current density $\left(J_{\mathrm{SC}}\right)$ in devices, several times larger than the values expected from bulk structures with similar material volumes [19]. Due to a much larger 
absorption cross-section (than the physical bounds), large absorption efficiency (>1) can be obtained in the vertical configuration [18] as manifested by the demonstration of a free standing GaAs NWSC with an extremely high current density exceeding $180 \mathrm{~mA} / \mathrm{cm}^{2}$ leading to an efficiency of $\sim 40 \%$ [16]. In addition to the aforementioned absorption characteristics due to the NW cavity, it is also obvious that the absorption along the length of the NW in a vertical NW solar cell is much different from that in a horizontal one, with the former being largely position dependent whereas the latter being uniform along the length of the NW. Therefore, for the horizontal NW solar cell with a simple axial p-n junction, with uniform absorption along axial direction the device p-n junction can be designed and optimized separately from the absorption property, providing a basic platform to understand the complicated behavior of nanoscale photovoltaic devices. So far single horizontal NW solar cells based on III-V materials have only been demonstrated in radial junction GaAs [16], InP [20], GaAsP [17] and GaN/InGaN/GaN [21] NWs. Compared to other III-V NWs, InP NWs have the advantage of very low surface recombination velocity (SRV) [2, 22-24] favorable for solar cell applications. An SRV value as low as $161 \mathrm{~cm} / \mathrm{s}$ has been achieved recently by our group for undoped and unpassivated InP NWs grown by SA-MOVPE [2]. However there has been very limited report on single axial junction InP nanowire solar cells [25].

Furthermore, dielectric materials such as silicon nitride $\left(\mathrm{SiN}_{\mathrm{x}}\right)$ have been widely used as antireflection (AR) coating and surface passivation layers for conventional planar Si solar cells [26] and InP-based bipolar transistors [27]. Theoretical and experimental studies also show that dielectric shells on NWs with subwavelength diameters can double light absorption due to the enhanced optical antenna effect [19]. For $\mathrm{Si}$ nanowires, the simulated results showed $\mathrm{SiN}_{\mathrm{x}}$ coating layer is more effective than $\mathrm{SiO}_{\mathrm{x}}$ coating layer due to its higher refractive index (i.e., $\sim 2$ compared to $\sim 1.46$ for $\mathrm{SiO}_{\mathrm{x}}$ at $633 \mathrm{~nm}$ ) $[19,28]$. On the other hand, despite the relatively low surface recombination velocity of InP NWs, it may be still crucial to passivate the surface of the 
nanowires to further improve the device performance. Simulations performed on InP nanowires revealed a 32\% improvement in efficiency when reducing the surface recombination [29]. Experimentally it has also been reported that the average efficiency of horizontal GaAsP single nanowire solar cells is significantly increased with the introduction of an epitaxially-grown InGaP passivation layer [17]. In this work, we present the fabrication and characterization of horizontally lying axial p-i-n junction InP single nanowire solar cells. The photovoltaic behavior of the devices has been investigated by a series of optical and electrical characterization and simulation techniques, to understand carrier generation, transport and collection processes. By further coating the NW devices with a conformal $\mathrm{SiN}_{\mathrm{x}}$ dielectric layer, the solar cell efficiency has been enhanced dramatically (up to $\sim 62 \%$ ) to $\sim 10.48 \%$, which has been ascribed to a combined optical antenna and surface passivation effect.

\section{Experimental Section}

\section{Nanowire growth}

The InP nanowire solar cell structure was grown in an AIXTRON 200/4 MOVPE reactor, operating at a base pressure of 100 mbar, using $\mathrm{H}_{2}$ as a carrier gas with a total flow of $14.5 \mathrm{l} / \mathrm{min}$. The axial p-i-n junction InP NW solar cell structures were grown by SA-MOVPE technique on a $\mathrm{SiO}_{2}$-masked $\mathrm{p}^{+}$-doped 111(A) InP substrate via electron beam lithography (EBL) and wet chemical etching (details see Ref. [2]). The substrate was designed to a pattern consisting of a hexagonal array of holes of $180 \mathrm{~nm}$ in diameter and $800 \mathrm{~nm}$ in spacing. The growth temperature was $730{ }^{\circ} \mathrm{C}$ with a V/III ratio of 80 . The flow rate for trimethylindium and phosphine was 6.07 $\times 10^{-6} \mathrm{~mol} / \mathrm{min}$ and $4.91 \times 10^{-4} \mathrm{~mol} / \mathrm{min}$, respectively. Silane and diethylzinc were used as $\mathrm{n}-$ and p-type dopants, with a flow rate of $3.05 \times 10^{-7} \mathrm{~mol} / \mathrm{min}$ and $2.03 \times 10^{-5} \mathrm{~mol} / \mathrm{min}$, respectively. The growth sequence for the NWSC structure was the p-segment first, followed by an undoped isegment and n-segment with a growth times of 10, 8, and 10 min, respectively. After growth, the 
NW array was imaged by scanning electron microscopy (SEM) and some NWs were transferred for examination by transmission electron microscopy (TEM).

\section{Single NW solar cell fabrication}

Single axial p-i-n junction InP NW solar cell devices were fabricated using EBL technique. Briefly, NWs were first mechanically transferred to a thermally oxidized $\mathrm{p}^{+}-\mathrm{Si}$ substrate with a 300-nm $\mathrm{SiO}_{2}$ layer. The electrodes were defined by EBL patterning and the resist residue on the NWs was removed using oxygen plasma by a barrel etcher. The NWs were then etched in a buffered HF solution to remove the surface native oxide. Finally Ti/Au (10/220nm) contacts were formed on each side of NWs using electron beam evaporation followed by a lift-off process.

\section{Single NW solar cell characterization}

The fabricated solar cells were characterized by current-voltage $(I-V)$ measurement in dark and under light illumination (1 Sun @ AM1.5G). The spectral response of the solar cells was measured under the short circuit condition (zero external bias) using a conventional amplitude modulation technique including a tungsten-halogen lamp as a white illumination source, a mechanical chopper, an Acton SpectraPro ${ }^{\circledR} 2300$ i monochromator, a Stanford SR570 low-noise current pre-amplifier and a Stanford SR830 DSP lock-in amplifier. The incident power of the white illumination source at each wavelength was measured using a calibrated Si photodetector for the calculation of the device's external quantum efficiency. The 2-dimensional reflection and photocurrent mappings of the fabricated solar cells were carried out with a WITec alpha300S scanning microscopy system under an excitation laser wavelength of $532 \mathrm{~nm}$. The beam was focused using a 100×, NA0.9 objective lens, and then scanned across the sample using a piezodriven sample stage. The photocurrent was detected using a similar amplitude modulation 
technique as described above. The reflected light was measured simultaneously using the confocal microscope and a Si avalanche photo-diode detector. Electron beam induced current measurements were performed on the fabricated devices in a FEI Helios 600 NanoLab DualBeam focused ion beam system.

\section{Dielectric film deposition}

The $\mathrm{SiN}_{\mathrm{x}}$ coating was deposited by plasma enhanced chemical vapor deposition (PECVD) at $100{ }^{\circ} \mathrm{C}$ using $\mathrm{SiH}_{4}, \mathrm{NH}_{3}$ and $\mathrm{N}_{2}$ with a flow rate of 25, 11 and $980 \mathrm{sccm}$, respectively, The process pressure was set to $650 \mathrm{mTorr}$ and RF power to $20 \mathrm{~W}$. A silicon substrate was also coated during the PECVD process for ellipsometry measurement, where the $\mathrm{SiN}_{\mathrm{x}}$ film was confirmed to have a refractive index of $\sim 1.96$ (at $633 \mathrm{~nm}$ ) and thickness of $\sim 100 \mathrm{~nm}$.

\section{Photoluminescence measurements}

The NWs were excited using an Yb: YAG laser with pulse duration 300 fs, repetition rate 20.8 $\mathrm{MHz}$, frequency-doubled to a wavelength of $522 \mathrm{~nm}$ using a LBO crystal. A spot size of 0.36 $\mu \mathrm{m}$ (in radius) was estimated by vector diffraction calculation under a $100 \mathrm{x}$ (NA 0.75) focusing objective. A charge-coupled device array was used to record the photoluminescence (PL) spectrum with a spectrometer and a Picoharp 300 time-correlated single photon counting system was used to detect PL decay. The time-resolved PL intensity decay at peak wavelength was fitted by a single-exponential decay [2] to extract the minority carrier lifetime.

\section{Results and discussion}

\subsection{Material and device characterization of axial junction InP single nanowire solar cells}




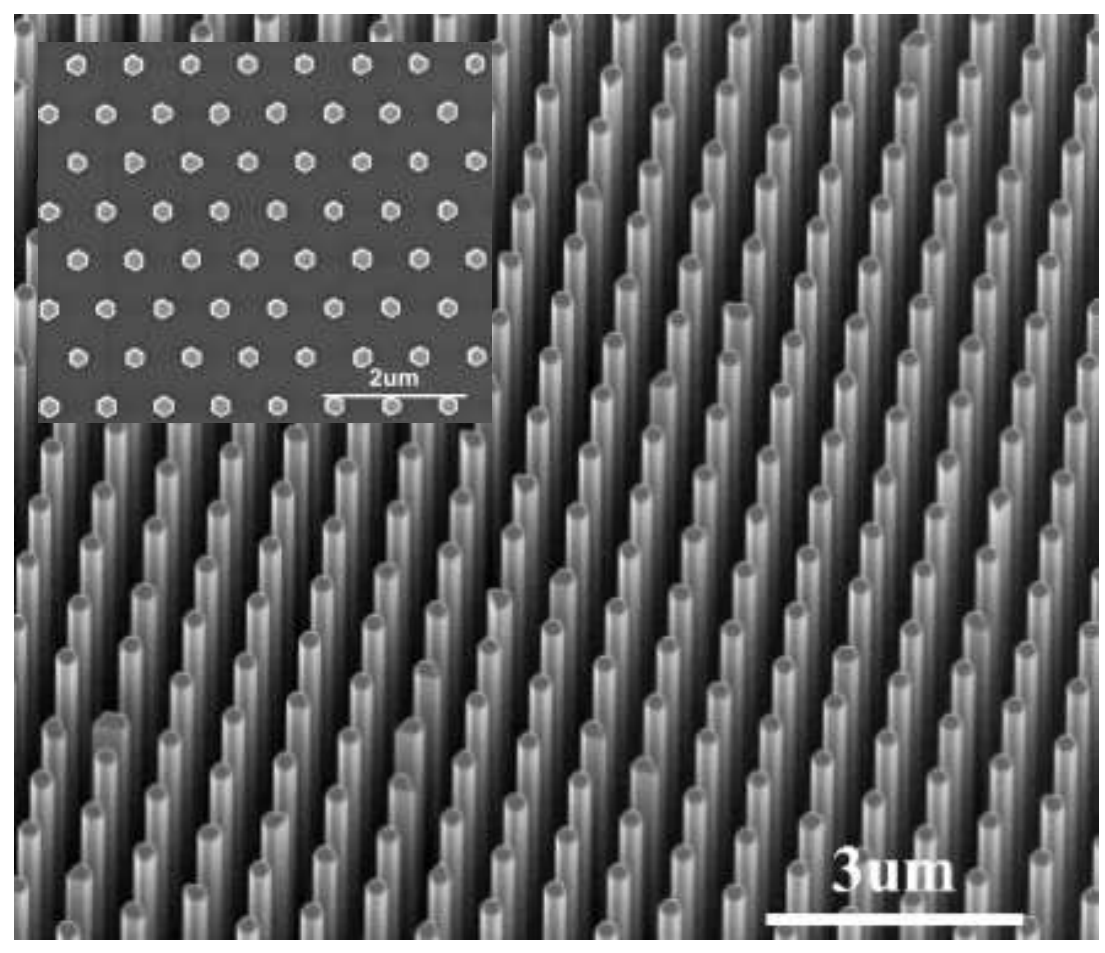

Fig. 1. SEM images at $30^{\circ}$ tilt view of the InP NW array grown for this work. Inset shows the top view SEM image.

An SEM image of the InP NW array grown for this study is displayed in Fig. 1 showing that the p-i-n InP NW array has a good surface morphology and relatively uniform lengths $(\sim 9-10 \mu \mathrm{m})$, however with some deviation from perfect hexagonal cross-section, which has also been observed previously in the undoped NWs due to the relatively large NW spacing $(800 \mathrm{~nm})$ and a consequent non-uniform lateral growth [2]. Nevertheless, the SAE related lateral growth does not seem to lead to the formation of a conformal radial junction, as confirmed later by the I-V characteristic in Fig. 2 which is a clear result of axial pn junction. TEM measurements of a batch of NWs $(>5)$ confirm that our NWs have a pure wurtzite crystal phase for all three (p, i, and n) regions (Supporting Information, Fig. S1) despite of their irregular shapes, similar to previously reported results from the undoped InP NWs [2]. Based on a detailed photoluminescence (PL) technique and electrical measurements [30] for NWs grown/doped under the same conditions, 

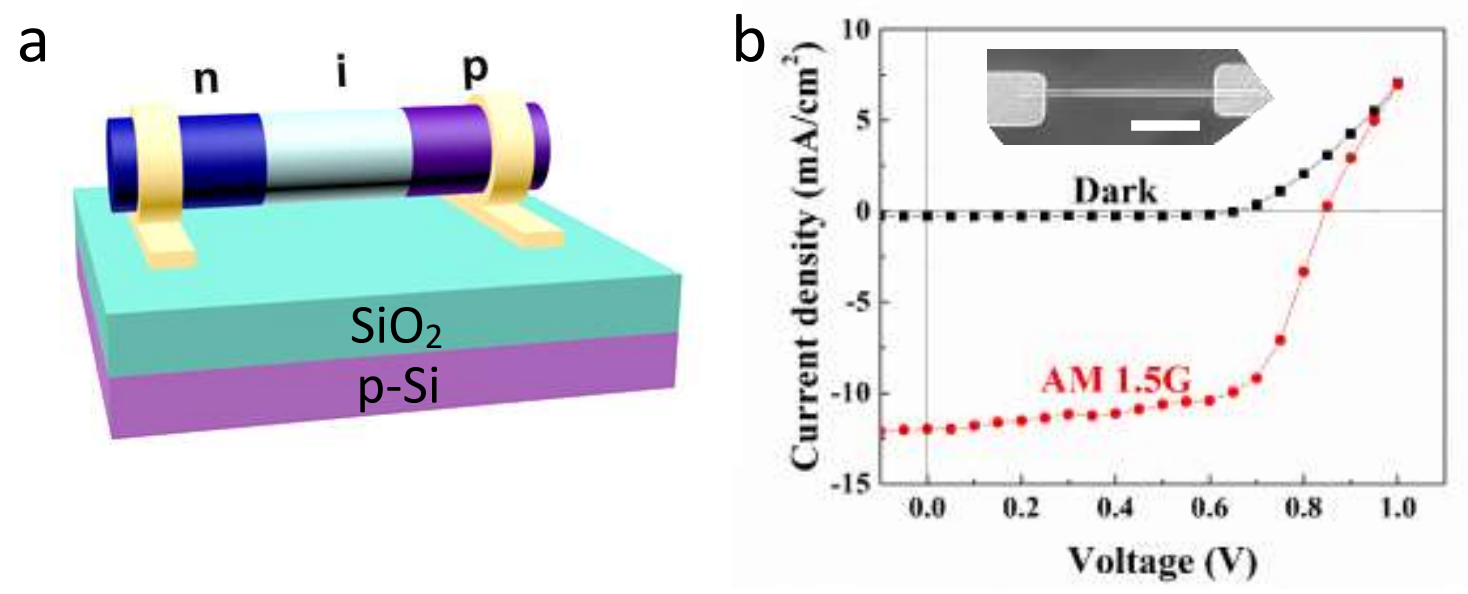

Fig. 2. (a) Schematic illustration of a single p-i-n InP NW solar cell. (b) $I-V$ characteristics under dark (black curve) and 1 Sun @ AM1.5G illumination conditions (red curve) of the fabricated solar cell. The inset shows SEM image of a fabricated single NW solar cell with the scale bar of $3 \mu \mathrm{m}$.

the doping concentration for n-region is $\sim 3 \times 10^{18} \mathrm{~cm}^{-3}$ and i-region is $\sim 1 \times 10^{17} \mathrm{~cm}^{-3}$ (with an ntype background doping). However despite the fact that a high doping concentration was aimed for the MOVPE growth of p-region, it appears to be difficult to determine the doping concentration using both PL and electrical measurements [30], indicating that the p-region of our NW is only lightly doped $\left(<1 \times 10^{17} \mathrm{~cm}^{-3}\right)$.

After the growth NWs were mechanically transferred to a thermally oxidized $\mathrm{p}^{+}-\mathrm{Si}$ substrate with a $300 \mathrm{~nm} \mathrm{SiO}_{2}$ layer and fabricated with $\mathrm{Ti} / \mathrm{Au}$ contacts on both ends of the NWs. The schematic illustration of a typical p-i-n InP NW solar cell device is shown in Fig. 2 (a), and the SEM image of a fabricated single p-i-n InP NWSC device is displayed in the inset of Fig. 2 (b). Fig. 2 (b) also shows the typical $I$ - $V$ characteristics measured from the NW devices under dark and 1 Sun@AM1.5G illumination conditions, exhibiting a clear photovoltaic behavior. Based 
on the SEM measurement of the projected area of the NW, a short circuit current density $\left(J_{\mathrm{sc}}\right)$ of $12 \mathrm{~mA} / \mathrm{cm}^{2}$, open circuit voltage $\left(V_{\text {oc }}\right)$ of $0.85 \mathrm{~V}$ and fill factor $(F F)$ of 0.64 is obtained from the

\begin{tabular}{|c|c|c|c|c|}
\hline & $V_{\text {oc }}(\mathrm{V})$ & $J_{\mathrm{sc}}\left(\mathrm{mA} / \mathrm{cm}^{2}\right)$ & $F F$ & $\eta(\%)$ \\
\hline Device 1 & 0.85 & 12 & 0.64 & 6.5 \\
\hline Device 2 & 0.88 & 9.1 & 0.64 & 5.2 \\
\hline Device 3 & 0.89 & 8.4 & 0.58 & 4.3 \\
\hline Device4 & 0.8 & 12.8 & 0.57 & 5.9 \\
\hline Device 5 & 0.82 & 6.7 & 0.63 & 3.5 \\
& & & & \\
\hline
\end{tabular}

(a)

\begin{tabular}{|c|c|c|c|c|}
\hline & $V_{\text {oc }}(\mathrm{V})$ & $J_{\text {sc }}\left(\mathrm{mA} / \mathrm{cm}^{2}\right)$ & $F F$ & $\eta(\%)$ \\
\hline Device 1 & 0.68 & 29.5 & 0.52 & 10.5 \\
\hline Device 2 & 0.57 & 28.6 & 0.64 & 10.4 \\
\hline Device 3 & 0.56 & 25.5 & 0.67 & 9.6 \\
\hline Device 4 & 0.56 & 28.6 & 0.56 & 9.0 \\
\hline Device 5 & 0.61 & 24 & 0.65 & 9.6 \\
\hline
\end{tabular}

(b)

Table 1. Summary of key performance parameters of (a) five InP single NWSCs and (b) five $\mathrm{SiN}_{\mathrm{x}}$-coated InP single NWSCs. 
device leading to a cell efficiency of $6.5 \%$. Table 1 (a) summarizes the key device parameters of five NWSCs. All these unpassivated InP devices exhibited excellent $V_{\text {oc }}$ ranging from 0.8 to 0.9 $\mathrm{V}$, which is comparable to the best reported values obtained from epitaxially passivated GaAsP/InGaP single NWs (horizontal) [17], indicating a high material quality of the SAMOVPE InP NWs [2]. However the $J_{\text {sc }}$ and $F F$ are comparable or slightly lower than the values obtained from the GaAsP/InGaP devices. Apart from the effects due to material bandgap, growth and device fabrication, the lower $J_{\text {sc }}$ could be due to two important reasons: 1) the reported GaAsP NW solar cells are based on a radial p-i-n junction which has the intrinsic advantage of efficient carrier collection due to the large junction area and short carrier collection path; and 2) for axial p-i-n NW solar cells the depletion region, contact resistance and thus the device properties are closely related to the doping concentration and length of the p-, i- and ntype regions, all of which are yet to be well understood and optimized. Therefore in this work, we employ nano-scale characterization techniques including 2D photocurrent mapping and EBIC measurement to reveal the spatial distribution of the photo- or electron- generated current to understand the electrical structure and performance of the NWSCs.

\subsection{Device analysis based on nano-scale characterization and simulation}

Figure 3 (a) presents the optical image of a single NW solar cell used for 2D photocurrent mapping measured under a $532 \mathrm{~nm}$ laser excitation at zero bias. As can be seen clearly, the spatial distribution of the photocurrent (as indicated by the white dotted circle in Fig. 3 (c)) corresponds very well to that of the un-contacted exposed part of the NW (as indicated by the white dotted circle in the reflection profile in Fig. 3 (b)), unambiguously confirming that the zero bias photocurrent originates from the NW itself, ruling out the possibility that photocurrent could also be generated at the metal/semiconductor contacts (if they are Schottky contacts). Combined with $I-V$ measurement, we confirm that a large photocurrent is generated from the 

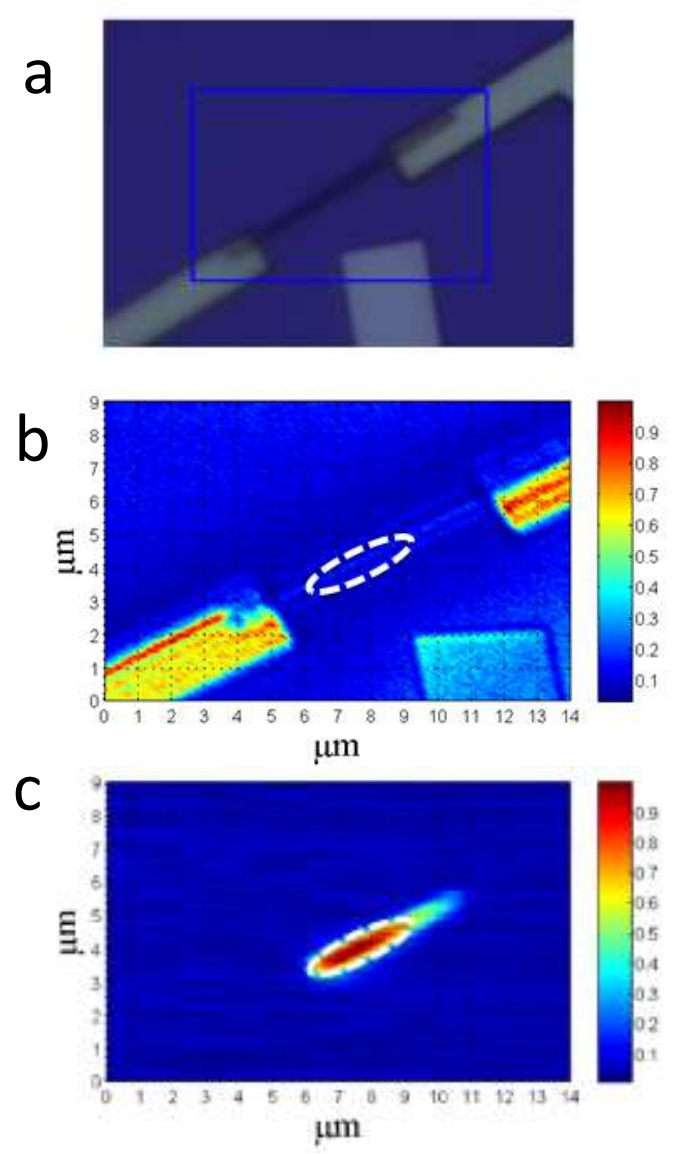

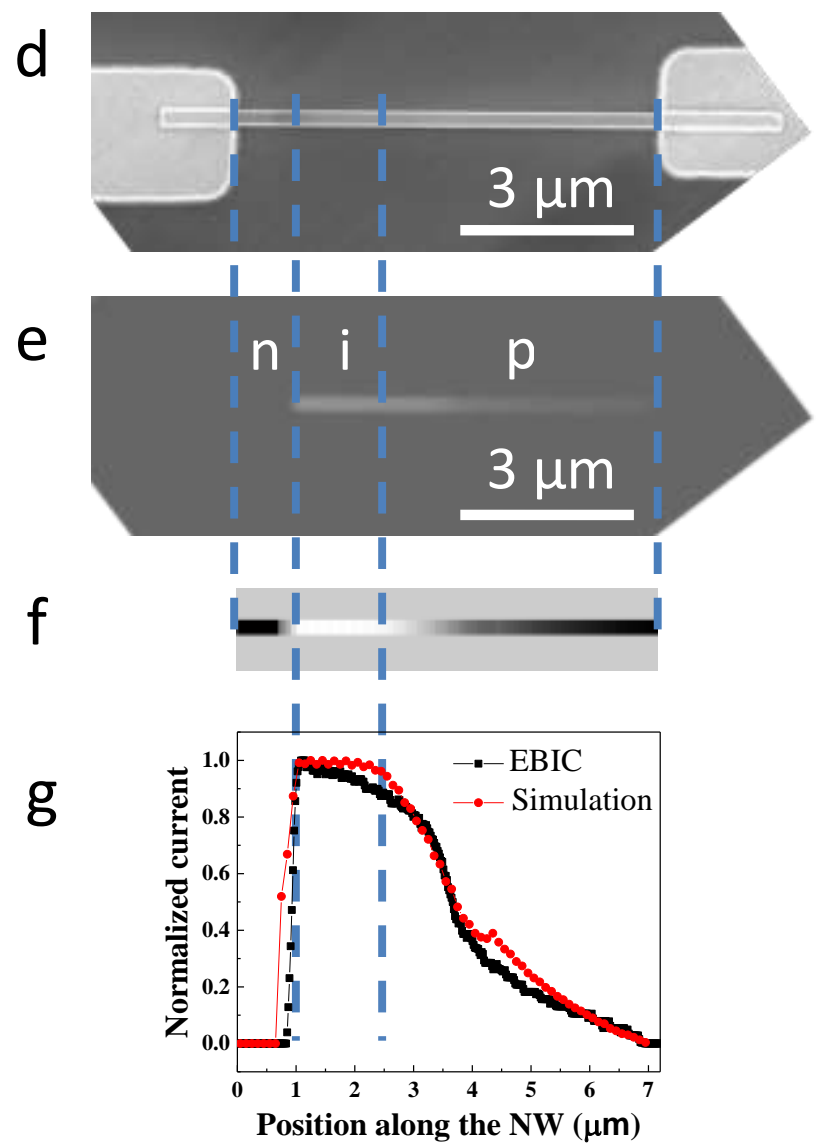

Fig. 3. (a) Optical image, (b) $2 \mathrm{D}$ reflection and (c) photocurrent mappings of a fabricated NW solar cell under $532 \mathrm{~nm}$ laser excitation with no bias voltage. (d) SEM image of a typical InP nanowire solar cell with the corresponding (e) EBIC response and (f) simulated current output based on Comsol. (g) EBIC and simulation line profiles along the center of the nanowire shown in (e) and (f), respectively.

region close to the $\mathrm{n}$-side, and is significantly reduced in the region close to the $\mathrm{p}$-side. About $0.7 \mu \mathrm{m}$ n-doped and $2 \mu \mathrm{m}$ p-doped regions show much reduced photocurrent signals, indicating the non-optimized device structure. In order to obtain a better spatial resolution for a deeper insight of the electrical structure of the single NW solar cell, EBIC measurements were performed on the fabricated devices with each side of the electrodes connected with the EBIC current input of the Helios FIB system. Using a highly focused electron beam ( sub-nanometer 
size) to excite the carriers and measure the short circuit current flowing in a material, EBIC is a common technique to identify p-n junction (width and position) and estimate minority carrier diffusion length in both planar photovoltaic devices [31, 32] and NWSCs [33, 34]. Fig. 3 (d) and (e) show the SEM image and the corresponding EBIC response for a representative nanowire device (> 10 devices have been measured with similar results). Fig. 3 (g) displays the EBIC current profile obtained at the wire center along the length of the nanowire. During the measurements, the NW devices were unbiased, therefore the EBIC signal originated from the separation of the electron beam generated nonequilibrium carriers by the built-in field of the junction as well as the diffusion of minority carriers from each side of the $p-n$ junction reaching the field. The EBIC image in Fig. 3 (e) displays three regions with clearly distinguishable contrasts, a totally dark region close to the n-contact, a very bright signal region in the middle, and a weaker signal region close to the p-contact. This result is consistent with the observation from the 2D photocurrent mapping however with a much improved spatial resolution, from which we assign 1) the region without EBIC signal to heavily doped n-segment $\left(\sim 3 \times 10^{18} \mathrm{~cm}^{-3}\right)$ due to the non-detectable hole diffusion current; 2) the region with bright EBIC signal to the iregion due to large built-in electric field and thus a large drift current; and 3) the weaker EBIC signal region to p-doped region which may be lightly doped leading to a detectable electron diffusion current. To understand further the doping profile in our NWSC structure and its effect on the junction formation and performance, we perform numerical simulation using Comsol Multiphysics (Semiconductor Module) to simulate the EBIC results (Supporting Information). By fixing the length of each segments, i.e., $\mathrm{n}=1 \mu \mathrm{m}, \mathrm{i}=1.5 \mu \mathrm{m}, \mathrm{p}=4.5 \mu \mathrm{m}$, and $\mathrm{n}$-doping concentration $\left(3 \times 10^{18} \mathrm{~cm}^{-3}\right)$, we vary the doping in the i-region and p-region to obtain a position dependent current output (Fig. 3 (f)) similar to that obtained from the EBIC measurement (Fig. 3 (e)). It can be seen that the simulated curve in Fig. 3 (g) is able to reproduce the main features exhibited in the EBIC result, when a low p-doping concentration of $1 \times 10^{15} \mathrm{~cm}^{-3}$ for the i-region 
and $1 \times 10^{16} \mathrm{~cm}^{-3}$ for the lightly doped p-region are assumed. It should be noted that assigning a low n-doping for the i-region will otherwise results in a very different current profile (see Fig. S2, Supporting Information). This is not surprising since $\mathrm{Zn}$ diffusion is a well-recognized problem during the growth of InP by MOVPE $[35,36]$. Due to the relatively high growth temperature of $730^{\circ} \mathrm{C}$ used for the NW growth, only low p-doping is obtained for the p-segment. In addition Zn may outdiffuse from the p-segment into the i-region with further growth (and thus lowering the p-type concentration even further). Some $\mathrm{Zn}$ may also be incorporated into the i-region during growth due to the "memory effect" in the reactor [37] since p-segment was grown firstly, making it slightly p-type. It is also expected that $\mathrm{Zn}$ diffusion may lead to a graded p-type doping concentration along the length of the NW, which could be the main reason for the small discrepancy between the simulated EBIC profile and the experimentally obtained one as shown in Fig. $3(\mathrm{~g})$.

Based on the 2D photocurrent mapping and EBIC measurement results, we believe that the efficiency of our NWSC can be further improved by a better structure design and growth optimization, including reducing the $\mathrm{n}$ - and $\mathrm{p}$-segment length, increasing the $\mathrm{p}$-segment doping concentration, and increasing the i-segment length. Reducing the $\mathrm{n}$ - and p-segment length and increasing the i-segment length can enhance the effective current density as the effective active area increases. Increasing the p-segment doping can definitely decrease series resistance and thus improve $V_{\mathrm{oc}}$ and $F F$. Due to the low diffusion current obtained from the lightly doped pregion, it is obvious that the device performance may not benefit greatly from having either a long $\mathrm{p}$ - or $\mathrm{n}$-segment. Indeed, for a horizontally lying axial $\mathrm{p}-\mathrm{i}-\mathrm{n}$ configuration, the main function for $\mathrm{p}$ - and n-type regions should be to provide the contacts to the junction embedded within the nanowire, hence their lengths should be kept as short as possible (provided it is feasible for fabrication). Also, there may be an optimum length of the i-region to maximize 
absorption while maintain sufficient built-in electric field strength for efficient carrier collection, which can be optimized through further simulation using Comsol Multiphysics.

\subsection{Characterization of SiN $_{x}$ coated InP nanowire solar cells}

To further investigate the effect of dielectric coating on III-V NWSCs, selected InP NW devices were deposited by a thin layer $(\sim 100 \mathrm{~nm})$ of $\mathrm{SiN}_{\mathrm{x}}$. The comparison of the $I-V$ characteristics under 1 sun illumination from the best devices with and without $\operatorname{SiN}_{x}$ coating layer is shown in Fig. 4. It can be seen that with the introduction of a $\mathrm{SiN}_{\mathrm{x}}$ layer, the device exhibits $V_{\text {oc }}, J_{\mathrm{sc}}, F F$ and efficiency of $0.68 \mathrm{~V}, 29.5 \mathrm{~mA} / \mathrm{cm}^{2}, 0.52$ and $10.5 \%$, respectively, with a significant increase

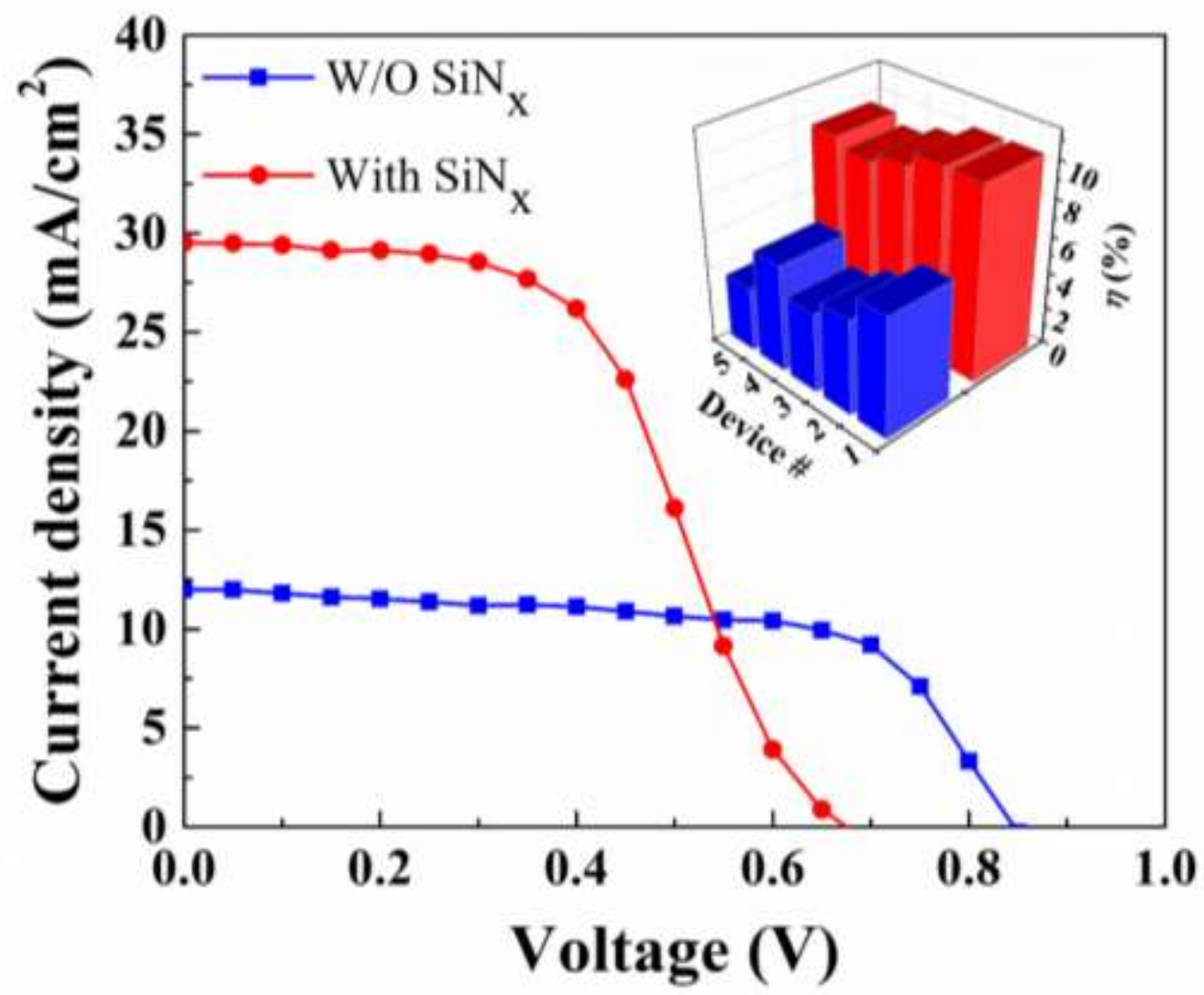

Fig. 4. $I-V$ characteristics under 1 Sun @ AM 1.5G illumination for the devices without (blue curve) and with (red curve) $\mathrm{SiN}_{\mathrm{x}}$ coating layer; Inset: Comparison of InP NWSC efficiencies without and with $\mathrm{SiN}_{\mathrm{x}}$ coating layer. 
of peak efficiency by $62 \%$ from the $6.5 \%$ of the best uncapped device. To our knowledge this is the highest efficiency value among the various types of horizontal single NW solar cells that have been reported so far $[16,17,21]$. Significant improvement of the overall conversion efficiency has been consistently observed in the $\mathrm{SiN}_{\mathrm{x}}$ coated devices, as demonstrated in the inset of Fig. 4 comparing the cell efficiencies of five $\mathrm{SiN}_{\mathrm{x}}$-coated devices with those of five uncoated ones. The large improvement in efficiency is mainly attributed to the striking enhancement of $J_{\mathrm{sc}}$, as shown in Table 1 (b) which summarizes the key performance parameters of five $\mathrm{SiN}_{\mathrm{x}}$-coated devices. However, while $J_{\mathrm{sc}}$ is improved by more than a factor of 2 after $\mathrm{SiN}_{\mathrm{x}}$ deposition without any obvious change in $F F$, a clear degradation of $V_{\text {oc }}$ is found in all the coated devices.

\subsection{Understanding the device performance enhancement by SiN $_{x}$ coating}

Improved current density from $\mathrm{SiN}_{\mathrm{x}}$ deposition may result from both enhanced optical antenna effect and surface passivation effect as mentioned earlier. The optical antenna effect by $\mathrm{SiN}_{\mathrm{x}}$ coating has been investigated theoretically and experimentally in horizontal radial junction single Si NWSCs, showing increase in $J_{\mathrm{sc}}$ as a direct result from broadband enhancement of the light-matter interaction between the incident plane wave and the optical resonator modes [38]. It is known that the waveguiding properties of nanowires arise due to the high index contrast between the nanowire and the surrounding medium. The absorption properties of the nanowires are a result of efficient coupling of incident light to the guided modes and confinement of the guided modes within the nanowire. Coating a dielectric shell around the nanowire affects both of these properties and hence changes the absorption characteristics of the NW [18]. Simulation results in photocurrent enhancement as a function of $\mathrm{SiN}_{\mathrm{x}}$ dielectric shell thickness for single, horizontally-oriented NW from Ref. [19] suggests that for InP NWs (with a similar diameter to our NWs) maximum enhancement can be achieved and reaches saturation at a coating 

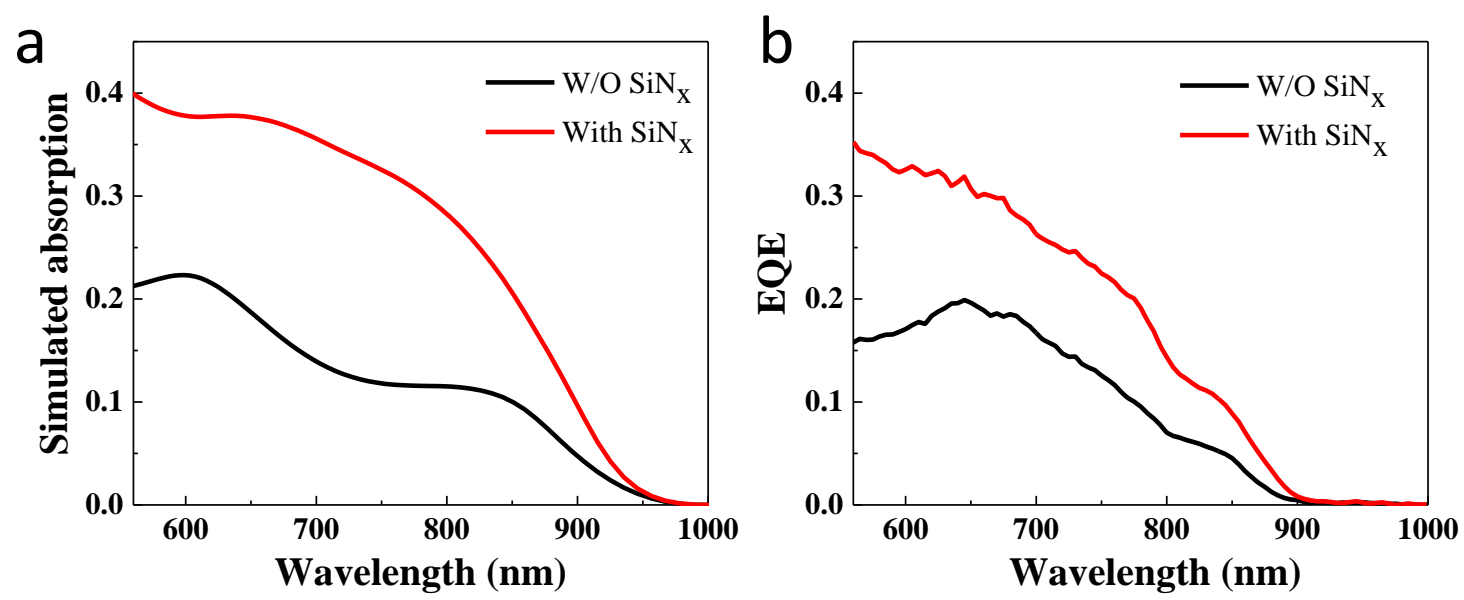

Fig. 5. (a) Simulated light absorption and (b) experimental EQE comparison for the NWs without and with a $\mathrm{SiN}_{\mathrm{x}}$ coating layer.

thickness of $\sim 100 \mathrm{~nm}$. To further confirm the optical absorption enhancement of the $\mathrm{SiN}_{\mathrm{x}}$ coating, FDTD simulation (Supporting Information) has been carried out to obtain the absorption efficiency spectrum of InP NW with a perfect hexagonal geometry and a side length of $180 \mathrm{~nm}$ (the typical side length of the NWs used in this work) for a NW with (100 nm) and without $\mathrm{SiN}_{\mathrm{x}}$ coating (see Fig. S3, Supporting Information). As expected, a broadband absorption enhancement with some variations of the number and position of the resonance peak after $\mathrm{SiN}_{\mathrm{x}}$ coating can be observed in Fig. S3. Furthermore, as mentioned earlier, it has been found that many of the InP NWs used in this study have irregular hexagonal shapes. To make sure the optical effect of $\mathrm{SiN}_{\mathrm{x}}$ coating are still valid, we perform the FDTD simulation on the light absorption in two of the best performed single NW solar cell devices (one with $\mathrm{SiN}_{\mathrm{x}}$ and one without), based on their exact shape as obtained by FIB cross-sectioning [39] (see Fig. S4, Supporting Information). As clearly displayed in Fig. 5 (a), for a NW with irregular hexagon shape but similar sizes, a much enhanced absorption can be obtained in the NW with dielectric coating. As a result, we observe a large enhancement of the experimentally measured external 

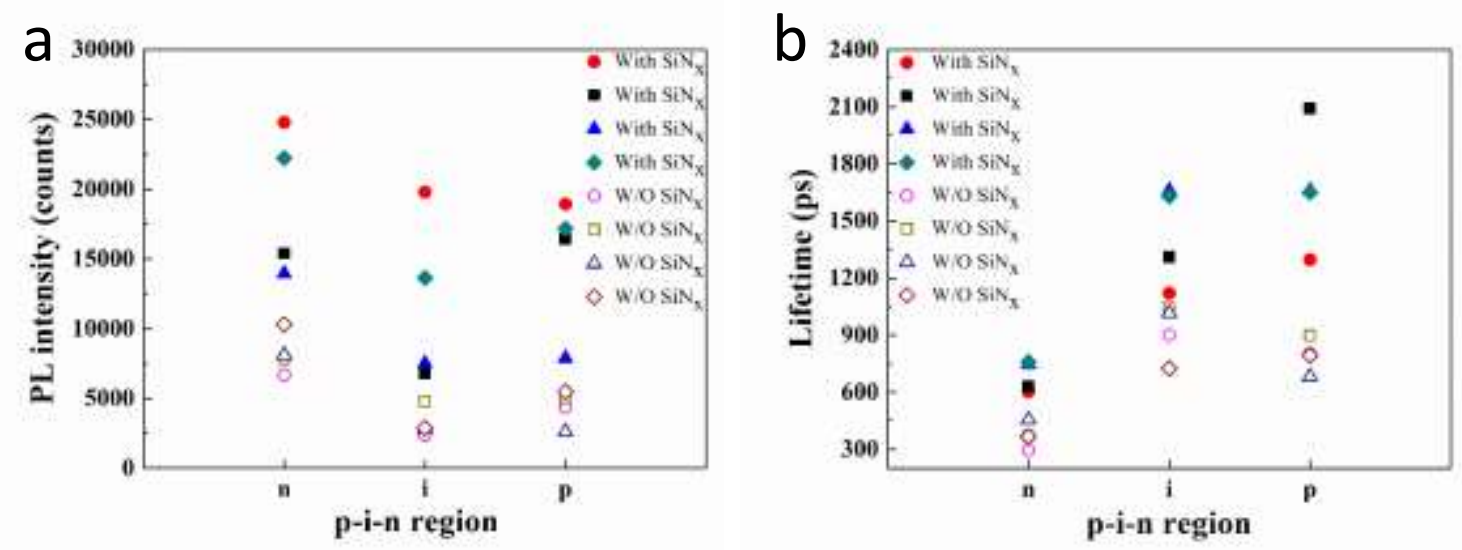

Fig. 6. Photoluminescence (a) Peak intensity and (b) lifetime comparison between the NWs with (solid) and without (open) $\mathrm{SiN}_{\mathrm{x}}$ coating layer.

quantum efficiency (EQE) from the $\mathrm{SiN}_{\mathrm{x}}$-coated NWSC in comparison to that from the uncoated one, as presented in Fig. 5 (b).

In addition to the absorption, a good surface passivation by $\operatorname{SiN}_{\mathrm{x}}$ coating could also contribute to the improved EQE. To investigate the passivation effect from $\mathrm{SiN}_{\mathrm{x}}$ coating we performed position dependent PL and TRPL measurements for the NWs without and with a $\mathrm{SiN}_{\mathrm{x}}$ coating layer (see Fig. S5 in the Supporting Information). Figure 6 summarizes the spatially-resolved PL peak intensity and minority carrier lifetime determined from four selected (from >10 measured devices) un-coated and $\mathrm{SiN}_{\mathrm{x}}$-coated $\mathrm{InP} \mathrm{NWs}$, respectively, showing that both the PL intensity and lifetime for all three regions ( $p, i, n)$ have been consistently increased after $\mathrm{SiN}_{\mathrm{x}}$ deposition. The PL intensity increase may be ascribed to both enhanced optical antenna effect and surface passivation effect from the $\mathrm{SiN}_{\mathrm{x}}$ coating; whereas the obvious lifetime increase in all $\mathrm{n}, \mathrm{i}$ and $\mathrm{p}$ regions is a clear indication of reduced carrier recombination, un-ambiguously confirming the passivation effect of the $\mathrm{SiN}_{\mathrm{x}}$ layer. However, it is worth mentioning that since the $\mathrm{SiN}_{\mathrm{x}}$ layer was deposited after the fabrication of single NWSC devices, 
the nanowire surface contacting with the underlying $\mathrm{SiO}_{2}$ was not coated with the $\mathrm{SiN}_{\mathrm{x}}$. $\mathrm{By}$ firstly coating the NW array with $\mathrm{SiN}_{\mathrm{x}}$ followed by a modified fabrication process [19], further improvement of device performance may be expected due to enhanced passivation and optical absorption in fully dielectric coated structure (in comparison to the partially coated one) [38].

Despite the significant photocurrent enhancement, one intriguing question remains for all the $\mathrm{SiN}_{\mathrm{x}}$-coated NWSCs is their reduced $V_{\mathrm{oc}}$. Normally with a good surface passivation, it is expected that $V_{\mathrm{oc}}$ will increase as a result of reduced surface recombination [38]. However it has been revealed from a detailed dielectric passivation study on Si nanowire solar cells [40], that a good surface passivation achieving a low surface recombination and good carrier lifetime do not necessarily result in a better solar cell performance. Even with a good chemical passivation, there may be a presence of surface fixed charge density due to the formation of dangling bonds or point defects at the passivation interface, resulting in large variation of carrier density and possible enhancement of the recombination rate and thus poor solar cell performance (especially lower $V_{o c}$ ). Therefore future study needs to be performed to understand the bonding configuration at the $\mathrm{SiN}_{\mathrm{x}} / \mathrm{InP}$ interface and confirm if any surface fixed charge density is formed to cause more recombination inside the NW and $V_{o c}$ degradation. Furthermore, it is known that standard PECVD deposition of $\mathrm{SiN}_{\mathrm{x}}$ is not a very desirable process for InP material, since the surface is bombarded by energetic ions that may create electronic defects [27] to affect the device performance. It is also found that non-optimized $\mathrm{SiN}_{\mathrm{x}}$ deposition conditions by PECVD may cause preferential etching of phosphorus on $\mathrm{InP}$ by $\mathrm{NH}_{3}$ plasma [41, 42], leading to degraded optical and electronic properties [27, 43, 44]. For bulk material plasma-induced damage mainly affects the region close to the surface, whereas for NWs the damage could have more significant effect on the whole absorbing region along the length of the NW that results in an overall increase of Shockley-Read-Hall recombination and a reduced $V_{\text {oc. }}$ A careful optimization of $\mathrm{SiN}_{\mathrm{x}}$ deposition conditions including the RF power, deposition temperature and 
gas flow rate may be required to minimize the surface fixed charge density and possible damage induced by PECVD deposition.

\section{Conclusions}

In conclusion, axial p-i-n junction InP nanowire solar cell structure was grown by SA-MOVPE technique. For the first time, unpassivated InP single NW solar cells with up to $6.47 \%$ efficiency have been demonstrated. By employing a series of nano-scale characterization techniques, we reveal the origin of the photocurrent in relation to the structural/electrical parameters of the axial $\mathrm{p}-\mathrm{i}-\mathrm{n}$ junction NWSC, as a critical guidance for future device optimization. Further coating of the NWSCs with $\mathrm{SiN}_{\mathrm{x}}$ layer has led to significant improvement of current density with enhanced efficiency to as high as $\sim 10.48 \%$. By combined optical simulation, photoluminescence and carrier lifetime measurements, the performance enhancement is attributed to enhanced optical antenna effect and surface passivation effect. Despite the evident surface passivation effect, an unusual reduction of $V_{\mathrm{oc}}$ after $\mathrm{SiN}_{\mathrm{x}}$ deposition is also observed and the possible reasons are discussed. Our work suggest that dielectric coating is a promising simple approach to enhance the performance of single InP nanowire solar cell performance and can be further extended to nanowire array solar cells, to achieve a wide range of next-generation low-cost high-efficiency devices.

\section{Appendix A: Supporting information}

\section{Acknowledgments}

We acknowledge the Australian Research Council (ARC) and National Natural Science Foundation of China (611760698) for financial support, and the Australian National Fabrication Facility (ANFF) ACT node for facility support. 


\section{References}

[1] D. Saxena, S. Mokkapati, P. Parkinson, N. Jiang, Q. Gao, H.H. Tan, C. Jagadish, Optically pumped room-temperature GaAs nanowire lasers, Nat Photon 7 (2013) 963-968.

[2] Q. Gao, D. Saxena, F. Wang, L. Fu, S. Mokkapati, Y. Guo, L. Li, J. Wong-Leung, P. Caroff, H.H. Tan, Selective-area epitaxy of pure wurtzite InP nanowires: high quantum efficiency and roomtemperature lasing, Nano letters 14 (2014) 5206-5211.

[3] S. Maeda, K. Tomioka, S. Hara, J. Motohisa, Fabrication and Characterization of InP Nanowire Light-Emitting Diodes, Japanese Journal of Applied Physics 51 (2012) 02BN03.

[4] E.D. Minot, F. Kelkensberg, M. Van Kouwen, J.A. Van Dam, L.P. Kouwenhoven, V. Zwiller, M.T. Borgström, O. Wunnicke, M.A. Verheijen, E.P. Bakkers, Single quantum dot nanowire LEDs, Nano letters 7 (2007) 367-371.

[5] V. Jain, A. Nowzari, J. Wallentin, M.T. Borgström, M.E. Messing, D. Asoli, M. Graczyk, B. Witzigmann, F. Capasso, L. Samuelson, Study of photocurrent generation in InP nanowire-based $\mathrm{p}^{+}-$ i- $n^{+}$photodetectors, Nano Research 7 (2014) 544-552.

[6] J. Wallentin, N. Anttu, D. Asoli, M. Huffman, I. Åberg, M.H. Magnusson, G. Siefer, P. FussKailuweit, F. Dimroth, B. Witzigmann, InP nanowire array solar cells achieving $13.8 \%$ efficiency by exceeding the ray optics limit, Science 339 (2013) 1057-1060.

[7] P. Parkinson, Y.-H. Lee, L. Fu, S. Breuer, H.H. Tan, C. Jagadish, Three-dimensional in situ photocurrent mapping for nanowire photovoltaics, Nano letters 13 (2013) 1405-1409.

[8] I. Aberg, G. Vescovi, D. Asoli, U. Naseem, J.P. Gilboy, C. Sundvall, A. Dahlgren, K.E. Svensson, N. Anttu, M.T. Bjork, A GaAs Nanowire Array Solar Cell With 15.3\% Efficiency at 1 Sun, Photovoltaics, IEEE Journal of 6 (2016) 185-190.

[9] Y. Cui, J. Wang, S.R. Plissard, A. Cavalli, T.T. Vu, R.P. van Veldhoven, L. Gao, M. Trainor, M.A. Verheijen, J.E. Haverkort, Efficiency enhancement of InP nanowire solar cells by surface cleaning, Nano letters 13 (2013) 4113-4117.

[10] X. Li, Y. Zhan, Enhanced external quantum efficiency in rectangular single nanowire solar cells, Applied Physics Letters 102 (2013) 021101.

[11] M. Yoshimura, E. Nakai, K. Tomioka, T. Fukui, Indium tin oxide and indium phosphide heterojunction nanowire array solar cells, Applied Physics Letters 103 (2013) 243111.

[12] X. Wang, M.R. Khan, M. Lundstrom, P. Bermel, Performance-limiting factors for GaAs-based single nanowire photovoltaics, Optics express 22 (2014) A344-A358.

[13] M.D. Kelzenberg, D.B. Turner-Evans, B.M. Kayes, M.A. Filler, M.C. Putnam, N.S. Lewis, H.A. Atwater, Photovoltaic measurements in single-nanowire silicon solar cells, Nano letters 8 (2008) 710-714.

[14] T.J. Kempa, B. Tian, D.R. Kim, J. Hu, X. Zheng, C.M. Lieber, Single and tandem axial pin nanowire photovoltaic devices, Nano letters 8 (2008) 3456-3460.

[15] N. Anttu, Shockley-Queisser Detailed Balance Efficiency Limit for Nanowire Solar Cells, ACS Photonics 2 (2015) 446-453.

[16] P. Krogstrup, H.I. Jørgensen, M. Heiss, O. Demichel, J.V. Holm, M. Aagesen, J. Nygard, A.F. i Morral, Single-nanowire solar cells beyond the Shockley-Queisser limit, Nature Photonics 7 (2013) 306-310.

[17] J.V. Holm, H.I. Jørgensen, P. Krogstrup, J. Nygård, H. Liu, M. Aagesen, Surface-passivated GaAsP single-nanowire solar cells exceeding $10 \%$ efficiency grown on silicon, Nature communications 4 (2013) 1498.

[18] S. Mokkapati, D. Saxena, H. Tan, C. Jagadish, Optical design of nanowire absorbers for wavelength selective photodetectors, Scientific reports 5 (2015) 15339.

[19] S.-K. Kim, X. Zhang, D.J. Hill, K.-D. Song, J.-S. Park, H.-G. Park, J.F. Cahoon, Doubling Absorption in Nanowire Solar Cells with Dielectric Shell Optical Antennas, Nano letters 15 (2014) 753-758.

[20] A. Nowzari, M. Heurlin, V. Jain, K. Storm, A. Hosseinnia, N. Anttu, M.T. Borgström, H.k. Pettersson, L. Samuelson, A Comparative Study of Absorption in Vertically and Laterally Oriented InP Core-Shell Nanowire Photovoltaic Devices, Nano letters 15 (2015) 1809-1814.

[21] Y. Dong, B. Tian, T.J. Kempa, C.M. Lieber, Coaxial group III- nitride nanowire photovoltaics, Nano letters 9 (2009) 2183-2187. 
[22] Y. Rosenwaks, Y. Shapira, D. Huppert, Metal reactivity effects on the surface recombination velocity at InP interfaces, Applied physics letters 57 (1990) 2552-2554.

[23] S. Münch, S. Reitzenstein, M. Borgström, C. Thelander, L. Samuelson, L. Worschech, A. Forchel, Time-resolved photoluminescence investigations on $\mathrm{HfO}_{2}$-capped InP nanowires, Nanotechnology 21 (2010) 105711.

[24] H.J. Joyce, J. Wong-Leung, C.-K. Yong, C.J. Docherty, S. Paiman, Q. Gao, H.H. Tan, C. Jagadish, J. Lloyd-Hughes, L.M. Herz, Ultralow surface recombination velocity in InP nanowires probed by terahertz spectroscopy, Nano letters 12 (2012) 5325-5330.

[25] M. Heurlin, P. Wickert, S. Fält, M.T. Borgström, K. Deppert, L. Samuelson, M.H. Magnusson, Axial InP nanowire tandem junction grown on a silicon substrate, Nano letters 11 (2011) 2028-2031.

[26] H. Nagel, A.G. Aberle, R. Hezel, Optimised antireflection coatings for planar silicon solar cells using remote PECVD silicon nitride and porous silicon dioxide, Progress in Photovoltaics: Research and Applications 7 (1999) 245-260.

[27] A. Ouacha, M. Willander, B. Hammarlund, R. Logan, Effect of surface passivation with SiN on the electrical properties of $\mathrm{InP} / \mathrm{InGaAs}$ heterojunction bipolar transistors, Journal of applied physics 74 (1993) 5602-5605.

[28] A. Solanki, P. Gentile, S. Boutami, V. Calvo, N. Pauc, Dielectric Coating-Induced Absorption Enhancement in Si Nanowire Junctions, Advanced Optical Materials 3 (2015) 120-128.

[29] S. Yu, F. Roemer, B. Witzigmann, Analysis of surface recombination in nanowire array solar cells, Journal of Photonics for Energy 2 (2012) 028002.

[30] F. Wang, Q. Gao, K. Peng, Z. Li, Z. Li, Y. Guo, L. Fu, L.M. Smith, H.H. Tan, C. Jagadish, Spatially Resolved Doping Concentration and Nonradiative Lifetime Profiles in Single Si-Doped InP Nanowires Using Photoluminescence Mapping, Nano letters 15 (2015) 3017-3023.

[31] P. Edwards, S. Galloway, K. Durose, EBIC and luminescence mapping of CdTe/CdS solar cells, Thin Solid Films 372 (2000) 284-291.

[32] L. Stolt, J. Hedström, J. Kessler, M. Ruckh, K.O. Velthaus, H.W. Schock, ZnO/CdS/CuInSe 2 thinfilm solar cells with improved performance, Applied Physics Letters 62 (1993) 597-599.

[33] P. Tchoulfian, F. Donatini, F. Levy, A. Dussaigne, P. Ferret, J. Pernot, Direct imaging of p-n junction in core-shell GaN wires, Nano letters 14 (2014) 3491-3498.

[34] P. Lavenus, A. Messanvi, L. Rigutti, A.D.L. Bugallo, H. Zhang, F. Bayle, F. Julien, J. Eymery, C. Durand, M. Tchernycheva, Experimental and theoretical analysis of transport properties of coreshell wire light emitting diodes probed by electron beam induced current microscopy, Nanotechnology 25 (2014) 255201.

[35] W. Tsang, F. Choa, N. Ha, Zinc-doping of InP during chemical beam epitaxy using diethylzinc, Journal of electronic materials 20 (1991) 541-544.

[36] F. Kellert, S. Sloan, M. Ludowise, J. Turner, Zn-doping in OMVPE Grown $\operatorname{lnP}: \mathrm{Zn} / \operatorname{lnGaAs} / \operatorname{lnP} p-i-$ $n$ double heterojunctlons with lnGaAs: $\mathrm{Zn}$ contacting layers, Journal of electronic materials 21 (1992) 983-987.

[37] E. Schubert, C. Pinzone, M. Geva, Phenomenology of Zn diffusion and incorporation in InP grown by organometallic vapor-phase epitaxy (OMVPE), Applied physics letters 67 (1995) 700-702.

[38] Y. Yu, V.E. Ferry, A.P. Alivisatos, L. Cao, Dielectric core-shell optical antennas for strong solar absorption enhancement, Nano letters 12 (2012) 3674-3681.

[39] L.A. Giannuzzi, F.A. Stevie, A review of focused ion beam milling techniques for TEM specimen preparation, Micron 30 (1999) 197-204.

[40] A.D. Mallorquí, E. Alarcón-Lladó, I.C. Mundet, A. Kiani, B. Demaurex, S. De Wolf, A. Menzel, M. Zacharias, A.F. i Morral, Field-effect passivation on silicon nanowire solar cells, Nano Research 8 (2015) 673-681.

[41] B. Lescaut, Y.I. Nissim, J.F. Bresse, Passivation of InGaAs surfaces with an integrated process including an ammonia DECR plasma, Eighth International Conference on Indium Phosphide and Related Materials (1996) 319-322.

[42] W. Ng, C. Tan, P. Houston, A. Krysa, A. Tahraoui, Surface passivation of InP/InGaAs heterojunction bipolar transistors, Semiconductor science and technology 19 (2004) 720.

[43] J. Kim, J.-H. CHA, M.-L. HA, C.-Y. KIM, Y.-S. KWON, RECVD silicon nitride passivation of InGaAsP with temperature ramping during deposition, Proceedings-Electrochemical Society (2005) 361-366. 
[44] A. Piccirillo, R. Marzano, A. Gobbi, P. Bagnoli, Photoluminescence investigation of III-V semiconductor surface damage induced by PECVD silicon nitride films, Applied surface science 52 (1991) 295-302. 